\title{
Toward Indoor Flying Robots
}

\author{
Jean-D. Nicoud ${ }^{1}$, Jean-C. Zufferey ${ }^{2}$ \\ ${ }^{1}$ DIDEL SA, Belmont, Switzerland,nicoud@didel.com \\ ${ }^{2}$ EPFL-I2S-ASL, Lausanne, Switzerland, jean-christophe.zufferey@epfl.ch
}

\begin{abstract}
Developing a research autonomous plane for flying in a laboratory space is a challenge that forces one to understand the specific aerodynamic, power and construction constraints. In order to obtain a very slow flight while maintaining a high maneuverability, ultralight structures and adequate components are required.
\end{abstract}

In this paper we analyze the wing, propeller and motor characteristics and propose a methodology to optimize the motor/gear/propeller system. The C4 model plane (50g, $1.5 \mathrm{~m} / \mathrm{s}$ ) demonstrates the feasibility of such a laboratory flying test-bed.

\section{Introduction}

After years of research on rolling and walking robots, why not moving to flying robots? The dynamics and thus the navigational possibilities and requirements of such vehicles are fundamentally different from terrestrial robots. This may lead not only to interesting behaviors but also to new kinds of controller schemes.

A number of projects already exist with airborne test-beds like remote controlled helicopters [1], planes (e.g. the military drones [2-3] or the well-known micro air vehicles, MAVs [4-7]), or airships but most of them are outdoor machines, consequently requiring large teams, considerable technical skills and significant budgets. It is to notice that very few of them are really autonomous devices.

For these reasons, doing outdoor flying robotics is too heavy for most of the research teams. Therefore, we propose to investigate the possibility of indoor flying. We believe that if an inexpensive laboratory flying test-bed is feasible, this field may become very appealing for many researchers.

The flying schemes can be classified into four categories: lighter-than-air, flapping wings, rotary wings, and fixed wings. All of them are not convenient for indoor use.
Airships or blimps are probably the easiest way to make a robot fly. The envelop size can be adapted to the payload (a $1 \mathrm{~m}$ diameter sphere filled with helium can approximately lift $100 \mathrm{~g}$ ). Powered with three or more DC motors, they are quiet and not dangerous. Provided with ingeniously arranged protections, they can bump into obstacles without damage. No in-depth knowledge in aerodynamics is required to build such a machine. Consequently, some robotic research teams already adopted this option [8-9]. The drawbacks of the blimp are its inertia associated with its quite important volume and the need for helium.

A flapping wings hummingbird is the dream for many researchers. Sustained flight has been demonstrated [10], but full control with a payload in an indoor environment is not for tomorrow. However, flapping wings represent the only hope to reduce the size below $10 \mathrm{~cm}$ wing span, and quite interesting projects are currently in progress [11], backed by serious fly studies [12].

Helicopters are most likely too dangerous and noisy. In general, $\mathrm{R} / \mathrm{C}$ helicopters tend to be heavy and expensive. However, the lightest indoor R/C helicopter [13] weighs only $50 \mathrm{~g}$, and even much smaller ones are considered by scientists [14]. But they may be too fragile, too sensitive to payload, and have very limited running time.

Hence it seems that autonomous indoor planes are an essential step toward the mastering of flying robots. The technology is partly available from indoor hobby plane suppliers and clubs (WES Technik, DIDEL, Aeronutz, RCmicroflight, Ezonemag).

This paper presents the essential elements for the modeling and design of an indoor fixed wings flying robot, with very low flight speed and adequate maneuverability for operation in a $10 x 10 \mathrm{~m}$ room. First some principles of basic aerodynamics are summarized and the correspondence laws are applied to get some clues on what happens with small dimensions and low speed. Then the wing, propeller, and motor design are tackled. Finally some thoughts about the weight distribution and the navigation control are given. 


\section{Basic Aerodynamics}

Lift and drag are generated by the wing or propeller moving in the air [15-16]. If $\rho$ is the air density (about $1.22 \mathrm{~kg} / \mathrm{m}^{3}$ at $20^{\circ} \mathrm{C}$, at sea level), $S$ the wing area and $v$ the relative air speed, then the lift $F_{L}$ and drag $F_{D}$ are given by

$$
\begin{aligned}
& F_{L}=\frac{1}{2} \rho C_{L} S v^{2} \cong 0.61 \cdot C_{L} S v^{2} \\
& F_{D}=\frac{1}{2} \rho C_{D} S v^{2} \cong 0.61 \cdot C_{D} S v^{2}
\end{aligned}
$$

The polar plots (figure 1) display the $C_{L}$ and $C_{D}$ parameters at different angle of attack. These coefficients also depend on the airfoil shape and the kind of airflow around it, which is determined by the Reynolds number $R e$ :

$$
R e=\frac{\rho L v}{\mu}=\frac{\rho v^{2}}{\mu v / L}=\frac{\text { inertia }}{\text { viscous }} \cong 68000 \cdot L v
$$

$\mu$ is the air dynamic viscosity $\left(1.8 \cdot 10^{-5} \mathrm{~kg} \cdot \mathrm{s}^{-1} \cdot \mathrm{m}^{-1}\right.$ at $\left.20^{\circ}\right)$ and $L$ a characteristic length of the airfoil (in general the wing chord). $R e$ represents the ratio of inertial to viscous forces.

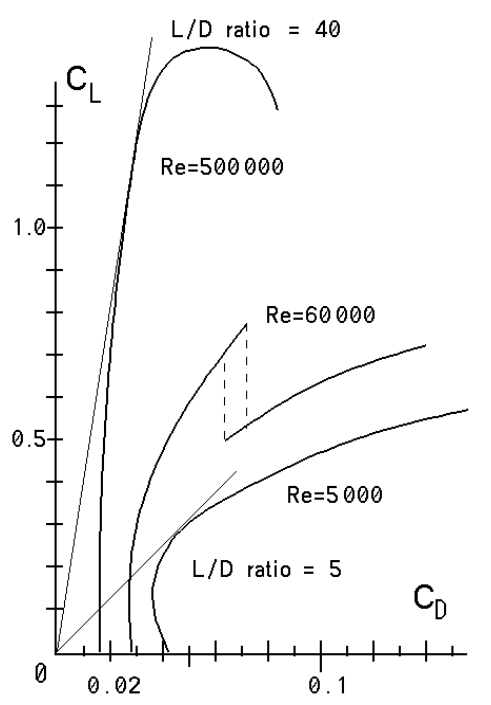

Figure 1: Typical polars for an airfoil at different Re

If the aerodynamic is good for large planes, it deteriorates rapidly for the small planes we are interested in [16-17]. Below a critical Re of about 50000, the viscosity influences the micro-turbulent flow against the surface, bubbles appear that break the lift, and the drag increases significantly. The lift to drag ratio (fineness), which is easily better than 40 for a modern glider, gets down to 3 to 5 for an indoor plane with a small aspect ratio. At $R e$ smaller than 1000, different aerodynamic principles have to be used: the flapping wings of hummingbird and insects create vortices on which the wings lean against [12].

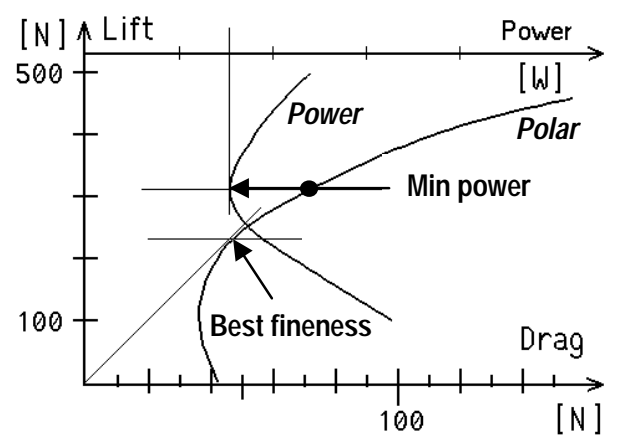

Figure 2: Best gliding and power ratio

In general, for indoor flying robots, the best lift/drag ratio is not exactly what we are interested in, but rather minimum power dissipation. This is the case if the ratio $\mathrm{F}_{\mathrm{L}}^{2} / \mathrm{F}_{\mathrm{D}}{ }^{3}$ is minimal [15]. Figure 2 shows the $\mathrm{C} 4$ model plane [18] polar measured in a wind tunnel at $1.5 \mathrm{~m} / \mathrm{s}$. In the same graph, the power curve is shown. Its minimum (in horizontal) gives the lift for minimum power.

For horizontal flight, the lift compensates exactly the weight $W\left(F_{L}=W\right)$. The wing loading $\sigma$ is an important parameter:

$$
\begin{aligned}
& \sigma=W / S \\
& W=F_{L} \cong 0.61 \cdot C_{L} \cdot S \cdot v^{2}
\end{aligned}
$$

For our plane, minimum power is required, in order to increase the flight time for a given battery.

$$
P=F_{D} \cdot v=0.61 \cdot C_{D} \cdot S \cdot v^{3}
$$

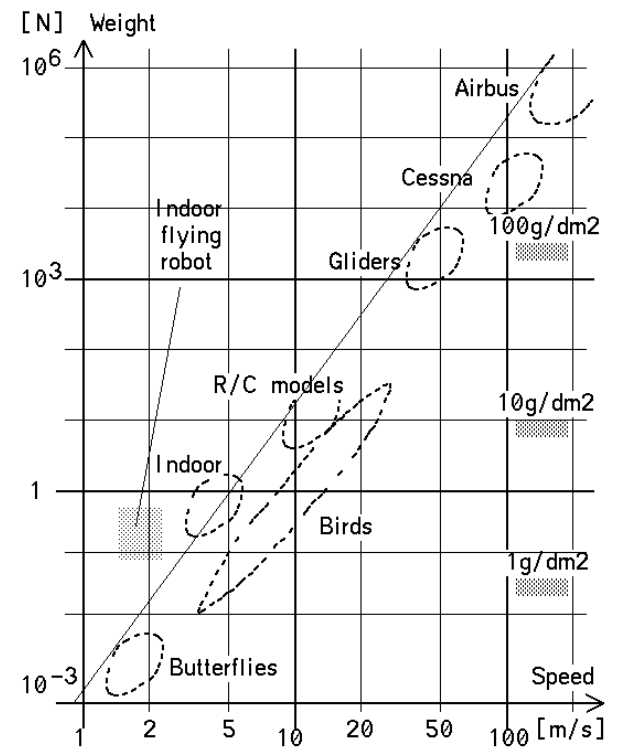

Figure 3: Aircraft weight vs speed

Flying at minimal power requires the lowest possible speed. This implies large wings and very low weight. 
What is realistic? Planes, birds and insects have developed minimal weight, reliable technologies, which are approximately plotted on figure 3 . We conclude that a $50 \mathrm{~g}, 1.5 \mathrm{~m} / \mathrm{s}$ plane is possible, but pushes the boundaries of the usual construction techniques.

For existing flying objects, $S$ and $v$ are linked by the plot of figure 3. The empirical value of the speed $v$ is related to the weight as follow:

$$
v \cong 5 \cdot \sqrt[4]{F_{L}}=5 \cdot \sqrt[4]{W}
$$

This formula does not result from similitude laws, but from nature evolution and engineers solutions taking care of manufacturing and energetic factors.

For a given weight, similitude analysis [19] shows that the lift $F_{L}$ is proportional to $S v^{2}$ (formula 1):

$$
F_{L} \propto S v^{2} \propto L^{2} v^{2}
$$

$L$ is a reference dimension and $v$ the speed. The drag is also proportional to $S v^{2}$ (formula 2), and the power $P$ for a horizontal flight is proportional to $S v^{3}$ (formula 6):

$$
P \propto L^{2} v^{3}
$$

For a given weight (formula 5):

$$
S \propto 1 / v^{2}, L \propto 1 / v, \text { or } v \propto 1 / L
$$

If the plane is twice as large for the same weight, it will fly two times slower.

We are more concerned with the power:

$$
P \propto L^{2} v^{3} \propto 1 / L
$$

The twice larger plane will need half the power, but again, this assumes the same weight.

\section{Wing Design}

For a $50 \mathrm{~g}$ indoor plane (included $20 \mathrm{~g}$ of payload for sensors and microcontroller), it is worth to stay below $3 \mathrm{~g} / \mathrm{dm}^{2}$ wing load, that is about $20 \mathrm{dm}^{2}(80 \mathrm{~cm}$ span and $26 \mathrm{~cm}$ chord length. Because of other heavy components (batteries, motors), the weight of the wing should be less than $5 \mathrm{~g}$. A good method for the construction of such a wing is to employ carbon rods for the frame and a thin plastic film for the cover, as used for the $\mathrm{C} 4$ model (figure 11). A realization that would respect a given documented airfoil is too heavy, supposing one has the data for that airfoil measured at the corresponding $R e$ number.

The shape of an ultra-light airfoil is given in figure 4. Gluing the film on the leading edge will result in burrs which may have a positive effect. Actually, the laminar flow is good for drag, but bubbles and then vortices will form easily with the lift suction. Creating some microturbulence is probably favorable, but extensive tests in a wind tunnel are still required to understand the phenomena and find the best light-weight shape.
It is not easy to find a wind tunnel with the very low speed we are interested in and having sensitive enough aerodynamic scales [17].

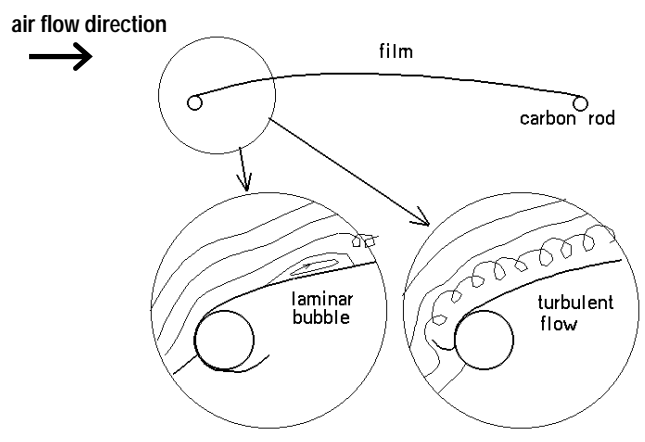

Figure 4: Lightweight airfoil

Computational models are promising, and may be of great help for the optimization of airfoils, which take care of the construction constraints. However, simple 2D airflow simulation programs (some of them are freely available on the internet) are not adapted for low $R e$ numbers.

\section{Propeller Design}

The theory for a propeller [15] gives the following similitude laws, which are easy to develop from a simplified model (Figure 5):

$$
\begin{array}{ll}
\text { Thrust } & T \propto S v^{2} \propto L^{2}(N L)^{2} \propto N^{2} L^{4} \\
\text { Torque } & M \propto S v^{2} L \propto N^{2} L^{5} \\
\text { Power } & P \propto S v^{2} L N \propto N^{3} L^{5} \\
\text { Reynolds number } & R e \propto v L \propto N L^{2}
\end{array}
$$

$N$ is the rotation speed (e.g. in RPM), and $L$ a reference dimension, e.g. the center of the blade.

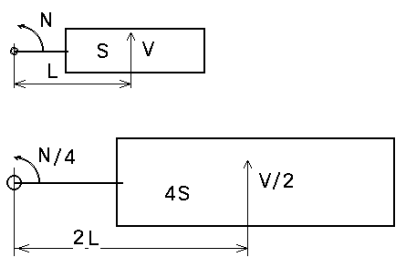

Figure 5: Propeller model

We are interested in getting a given thrust, and the challenge is to find the optimal blade dimensions. If $T \propto$ $N^{2} L^{4}$ is constant, then $N \propto 1 / L^{2}$. Hence,

$$
\begin{array}{ll}
\text { Power } & P \propto N^{3} L^{5} \propto 1 / L \\
\text { Reynolds number } & R e \propto N L^{2}=\text { constant }
\end{array}
$$

As a result, a twice larger propeller (figure 5) will spin at a quarter speed and require half the power. There is no aerodynamic change due to the $R e$ number, but the larger 
propeller cannot be built with the same technology (it would be too heavy, since the weight $\propto L^{3}$ ) and the aerodynamic parameters may change.

The theoretical shape for a propeller of a given pitch is easy to understand (figure 6). The pitch depends on the propeller's rotation speed and the plane's air speed. However, the air is pushed by the propeller at a speed that is difficult to know. We have plotted that speed for a steady plane (figure 6). The optimal angle of incidence for every section is hence difficult to define.

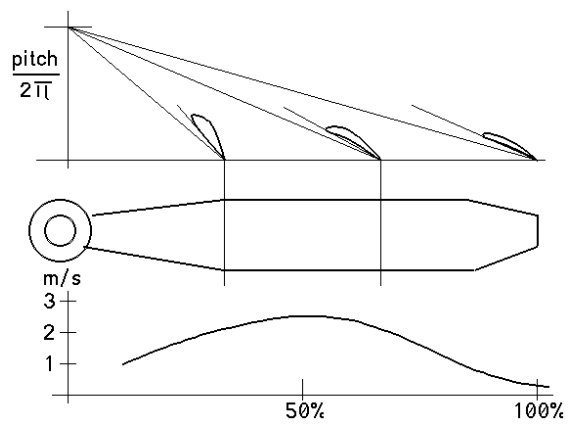

Figure 6: Propeller design and generated air-flow

Building balsa propellers is quite easy; testing them on a static bench with a balance gives a good idea of their quality at $1-2 \mathrm{~m} / \mathrm{s}$. Data are available from our web site [20]. Preliminary results (figure 7) show that "goodlooking" propellers of the same diameter have quite similar performances; their weight is related to their stiffness and maximum thrust. Commercial models are appropriate for $100-200 \mathrm{~g}$ model planes but too heavy for ultra-light indoor slow flyers.

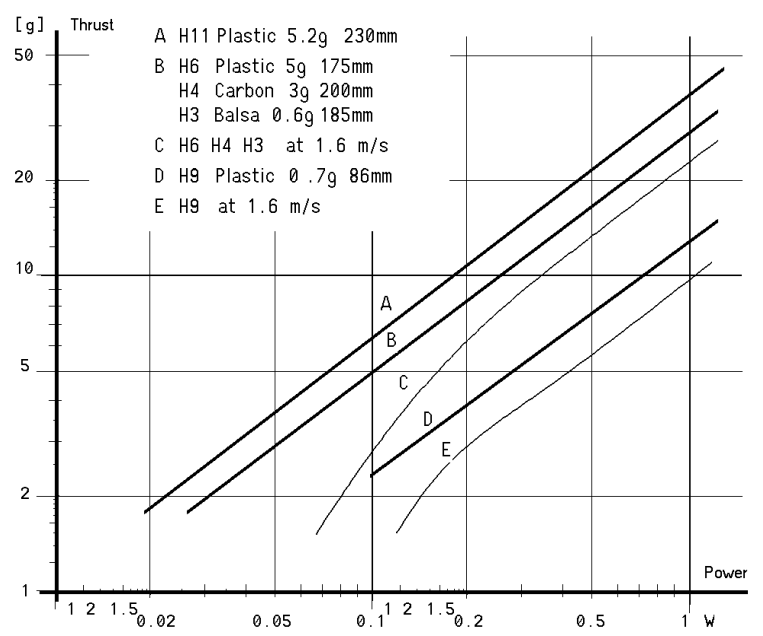

Figure 7: Propeller comparison

Notice that with relative air speed (measurements in wind tunnel), the thrust decreases at low torques. It even becomes negative when the torque is zero: the propeller is rotated by the wind - and has a higher drag than a blocked propeller.

Not surprisingly, the slope of the propellers lines is $2 / 3$, as predicted by equations (12) and (14).

\section{Motor Selection}

Brushless motors are the lightest and the most efficient, but they need bulky command electronics. Therefore the use of coreless DC motors is almost inevitable. Those are available from a diameter of $4 \mathrm{~mm}$ as low (pager motors) or high quality motors. These motors have a quite linear characteristic (figure 8).
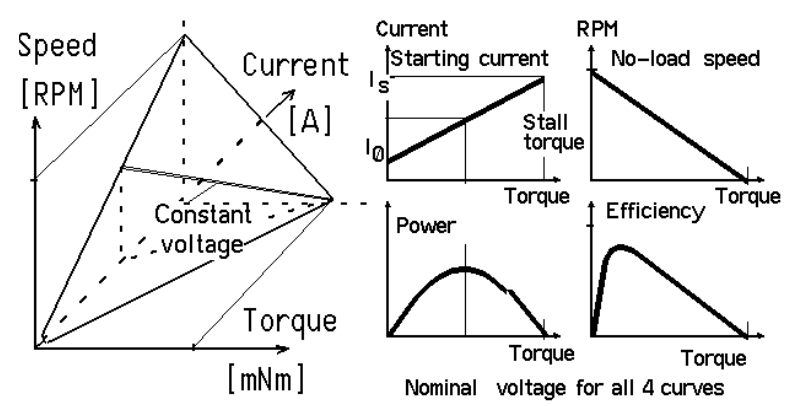

Figure 8: DC motor characteristics

The torque is proportional to the current I (torque constant $\mathrm{k}$ ), and the rotation speed induces an electromotive force (EMF) that reduces the consumed current.

For a given motor, the power depends on the voltage, and significant over-voltage is possible if heat is well dissipated. Lifetime will be inversely proportional to the power. The coil resistance $R$ dissipates power, and the power to weight efficiency depends on the magnet force, the coil volume and the air gaps.

$$
\begin{array}{ll}
\text { Torque } & M=k I \\
\text { Electrical power } & P_{e l}=U I \\
\text { Mech. power } & P_{m e c}=\omega M=2 \pi(N / 60) k I \\
\quad=P_{e l}-\text { losses }=U I-R I^{2}-\text { friction losses }
\end{array}
$$

$U$ and $I$ are the voltage and current supplying the motor. $\omega$ is the rotation speed $\left[\mathrm{s}^{-1}\right]$ and $I_{0}$ the no-load current. Note that the ratio between $I_{s}$, the stall current, and $I_{0}$ gives a good idea of the quality of the motor.

Maximum power is obtained at half the maximum torque, with half the maximum current:

$$
\begin{aligned}
P_{\text {el,max }} & =U I_{\max }=U U /(2 R)=U^{2} /(2 R) \\
P_{\text {mec, } \text { max }} & =U^{2} /(2 R)-R I_{\text {max }}{ }^{2} \\
& =U^{2} /(2 R)-R\left[U /(2 R)-I_{0}\right]^{2} \approx U^{2} /(4 R)
\end{aligned}
$$

Adding a gear will increase the torque and reduce the output rotation speed, allowing better match with the propeller, as shown in figure 9. 
Associated with the motor curves are current values that will define the battery size. Associated with the propeller curves are thrust values. A minimum thrust of about one third of the airplane's total weight is required for horizontal flight. Matching an existing propeller to a motor for which the reduction factor can be selected is hence possible. Reduction gears of different ratios are available for 4 to $10 \mathrm{~mm}$ motors [20].

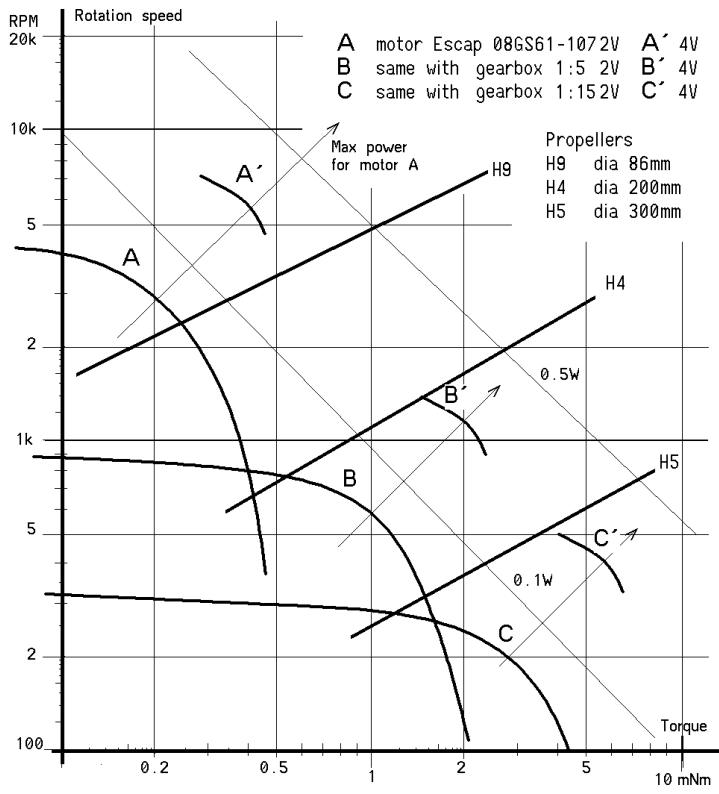

Figure 9: Motor and propeller power

\section{Weight Budget}

The problem with indoor flying is the weight of the batteries, reaching easily one third of the overall weight of the plane. Battery choices are limited for a model weighing less than $50 \mathrm{~g}$. The smallest NiMH (1/3AAA format from $\mathrm{GP}, 1.2 \mathrm{~V}, 70 \mathrm{mAh}$ ) weighs $2.4 \mathrm{~g}$ per cell and has an internal resistance of $50 \mathrm{~m} \Omega$. This means that a 4cells pack will deliver only $500 \mathrm{~mA}$ at $4 \mathrm{~V}$, one third of the battery power being dissipated within the battery itself. Lithium-Ion batteries (e.g., Renata, 330mAh, 10.1g) seem to be a good solution for improved endurance but require a step-up converter since their nominal output voltage is only $3.7 \mathrm{~V}$. Additionally, great care must be taken for the charging procedure. More recently, Lithium-Polymer batteries (e.g., 3.7V, 135mAh, 3.5g) have appeared on the market. They are quite lightweight since no hard package is needed.

Servos are also rather heavy. As a reference, the WES $2.4 \mathrm{~g}$ servo has a force of $150 \mathrm{~g}$, and a current consumption of $100 \mathrm{~mA}$ (which both are too high for our purposes). Magnets-in-a-coil servos are lighter but have a considerably reduced force. For savings of at least one servo and increasing maneuverability, a twin-motor plane is a solution to be tested. Motor speed control costs only a $0.1 \mathrm{~g}$ transistor, but two motors instead of one is not the best for efficiency.

Off-the-shelf radio control receivers are available to as light as 2.3g. Infrared controls represent another unidirectional communication possibility for indoor flights, which weighs a bit less. We are currently investigated a digital bidirectional radio solution. The first prototype weighs about $4 \mathrm{~g}$, included a microcontroller for onboard control, a speed controller, and a step-up DC-DC converter.

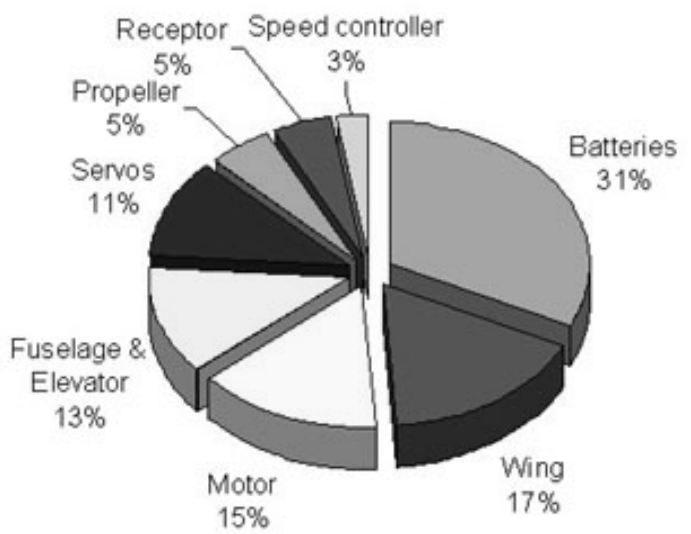

Figure 10: Weight budget for the $50 \mathrm{~g} C 4$ model plane

Considerable care must be taken to reduce the weight everywhere. For the $\mathrm{C} 4$ plane, the weight budget is given in figure 10, and shows that half of the weight is in the batteries, servos and radio accessories.

\section{Sensors, Navigation and Control}

In addition to the weight budget of figure 10, a robot needs autonomous navigation components, which correspond at present to rather bulky electronics on "standard" mobile robots. Miniature electronic components and microcontrollers are quite easily available, but sensors are usually encapsulated without consideration of the weight. A one dimensional range finder may be useable for altitude control, but it is unconceivable to mount a sufficient number of distance sensors for general obstacle avoidance.

Inspiration should rather be taken from insects. For example, flies have compound eyes with very coarse resolution that indeed enable them to efficiently navigate through cluttered environments. Hence, it must be possible to obtain an obstacle avoidance behavior by using basic vision sensors [21-22] with few pixels or photoreceptors, and thus a low power requirement. 


\section{Conclusion}

In this paper, we analyzed the different key components of a laboratory fixed-wing flying robot, namely its aerodynamics (wing), and its propulsion system (DC motor, gear, and propeller). We found that (i) aerodynamics at low Reynolds number is critical but still good enough for our purposes, (ii) some simple building techniques exist, which allow for realizing such a plane, (iii) there is a theoretical way to optimize the motor/gear/voltage/propeller set.

In order to demonstrate in real-life the conclusions of this analysis, a remote controlled model [18] has been developed, which is able to fly as slow as $1.4 \mathrm{~m} / \mathrm{s}$ (without payload). It weighs $47 \mathrm{~g}$ and can easily maneuver in a $10 \times 10 \mathrm{~m}$ room. It uses an original solution in order to improve maneuverability at low speed - the direction is controlled by rotating the thrust system (motor, reduction gear, and propeller) around a vertical axis. This allows for tight turns with a radius of about $2 \mathrm{~m}$.

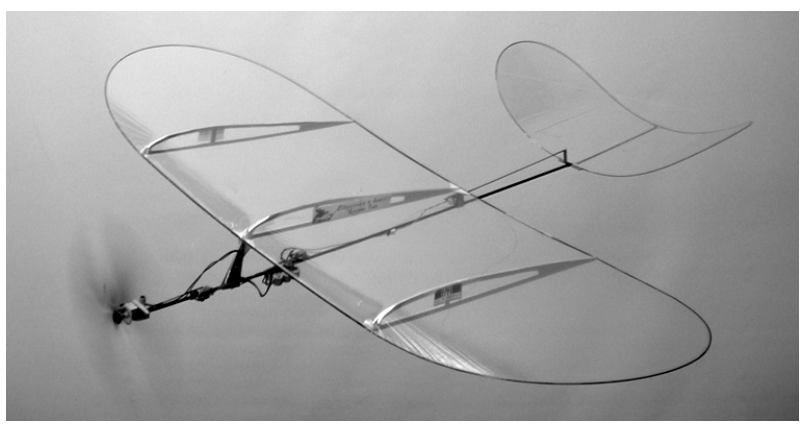

Figure 11: Model C4

Hopefully this work will contribute to the expansion of the indoor flying robot research field. Actually, we believe it is an appropriate and attractive test-bed for the development of bio-inspired robot controllers.

\section{Acknowledgements}

We would like to especially thank Cyril Halter, who significantly contributed to the construction of the $\mathrm{C} 4$ and to the aerodynamic analyses. We are also indebted to R. Hertig and D. Floreano at EPFL who provided the wind tunnel and technical support, and with whom we had number of motivating discussions.

Jean-C. Zufferey was supported by the Swiss National Science Foundation, grant nr. 620-58049.

\section{References}

[1] "Autonomous flying helicopter", www.heli.ethz.ch

[2] M. Hewish, "Building a bird's.eye view of the battlefield", Janes International Defense Review, No 2, 1997, pp55-62.
[3] R.G. Austin, "The Sprite aerial robot", Industrial Robot, Vol 24 No 2, 1997, pp152-157.

[4] J.M. McMichael, M.S. Francis, "Micro Air Vehicles Toward a New Dimension in Flight", www.darpa.mil/tto/MAV/mav_auvsi.html

[5] “TS6. Micro air vehicles", www.ukdf.org.uk/ts6.html

[6] J.M. Grassmeyer, M.T. Keennon, "Development of the Black Widow Micro Air Vehicle", AIAA-2001-0127, www.aerovironment.com/area-aircraft/prod-serv/ bwidpap.pdf

[7] S.J. Morris, M. Holden, "Design of Micro Air Vehicles and Flight Test Validation, MLB Company, 2000, www.spyplanes.com/Background/notredame0600.pdf

[8] F. Iida (2001), "Goal-Directed Navigation of an Autonomous Flying Robot Using Biologically Inspired Cheap Vision", Proceedings of the 32nd International Symposium on Robotics.

[9] H. Zhang, J.P. Ostrowski (1998), "Visual Servoing with Dynamics: Control of an Unmanned Blimp", T. report.

[10] T.N. Pornsin, Y.C. Tai, M. Keennon, (2001). "Microbat: A Palm-Sized Elecrically Powered Ornithopter", touch.caltech.edu/publications/2001/jpl/jpl2001.pdf

[11] R.S. Fearing, K.H. Chiang, M.H. Dickinson, D.L. Pick, M. Sitti, and J. Yan (2000), "Wing Transmission for a Micromechanical Flying Insect". IEEE Int. Conf. Robotics and Automation, robotics.eecs.berkeley.edu/ ronf/mfi.html

[12] M.H. Dickinson, F.O. Lehmann, S.P. Sane (1999), "Wing Rotation and the Aerodynamic Basis of Insect Flight", In: Science, vol 284, pp. 1954-1960, socrates.berkeley.edu/ flymanmd/

[13] A. Van de Rostyne "Pixel gallery: Aerodynamics and technology", www.planetinternet.be/pixel/

[14] A. Kroo, "The Mesicopter: A Meso-Scale Flight Vehicle", 1999, aero.stanford.edu/mesicopter

[15] R. von Mises, "Theory of Flight", Dover publications, New York, 1959, 630p.

[16] M. Simons, "Model Aircraft Aerodynamics", Argus Book, London, 1987, 318p.

[17] A. Pelletier, T.J. Mueller, "Aerodynamic Force/Moment Measurements at Very Low Reynolds Numbers", Proceedings from the 46th Annual Conference of the Canadian Aeronautics and Space Insitute, Montreal, May 35, 1999, pp. 59-68.

[18] J.C. Zufferey, C. Halter, J.D. Nicoud, “Avion d'intérieur: une plate-forme de recherche pour la robotique bioinspirée", Modell Flugsport No6, 2001, pp5-10, dmtwww.epfl.ch/isr/east/team/zufferey/project_isf.html

[19] I. Shimoyama, "Scaling in Microrobots", Proceedings IROS'95, Pittsburgh, August 1995, pp 208-211.

[20] J.D. Nicoud, "Propeller tests", www.didel.com/slow/ propellers/

[21] Franceschini, N., Pichon, J.M., Blanes, C. "From insect vision to robot vision", Phil. Trans. R. Soc. B337, 283-294, 1992

[22] Neumann, T.R. and H.H. Bülthoff, "Insect inspired visual control of translatory flight", Advances in Artificial Life Proceedings of the 6th European Conference on Artificial Life ECAL 2001 